\title{
Development and validation of an algorithm to recalibrate mental models and reduce diagnostic errors associated with catheter-associated bacteriuria
}

Barbara W Trautner ${ }^{1,2,4}$, Rupal D Bhimani ${ }^{2}$, Amber B Amspoker ${ }^{1,2}$, Sylvia J Hysong 1,2, Armandina Garza ${ }^{1,2}$, P Adam Kelly ${ }^{3}$, Velma L Payne ${ }^{1}$ and Aanand D Naik ${ }^{1,2^{*}}$

\begin{abstract}
Background: Overtreatment of catheter-associated bacteriuria is a quality and safety problem, despite the availability of evidence-based guidelines. Little is known about how guidelines-based knowledge is integrated into clinicians' mental models for diagnosing catheter-associated urinary tract infection (CA-UTI). The objectives of this research were to better understand clinicians' mental models for CA-UTI, and to develop and validate an algorithm to improve diagnostic accuracy for CA-UTI.

Methods: We conducted two phases of this research project. In phase one, 10 clinicians assessed and diagnosed four patient cases of catheter associated bacteriuria ( $n=40$ total cases). We assessed the clinical cues used when diagnosing these cases to determine if the mental models were IDSA guideline compliant. In phase two, we developed a diagnostic algorithm derived from the IDSA guidelines. IDSA guideline authors and non-expert clinicians evaluated the algorithm for content and face validity. In order to determine if diagnostic accuracy improved using the algorithm, we had experts and non-experts diagnose 71 cases of bacteriuria.

Results: Only 21 (53\%) diagnoses made by clinicians without the algorithm were guidelines-concordant with fair inter-rater reliability between clinicians (Fleiss' kappa $=0.35,95 \%$ Confidence Intervals $(C l s)=0.21$ and 0.50 ). Evidence suggests that clinicians' mental models are inappropriately constructed in that clinicians endorsed guidelines-discordant cues as influential in their decision-making: pyuria, systemic leukocytosis, organism type and number, weakness, and elderly or frail patient. Using the algorithm, inter-rater reliability between the expert and each non-expert was substantial (Cohen's kappa $=0.72,95 \% \mathrm{Cls}=0.52$ and 0.93 between the expert and non-expert \#1 and $0.80,95 \% \mathrm{Cls}=0.61$ and 0.99 between the expert and non-expert \#2).

Conclusions: Diagnostic errors occur when clinicians' mental models for catheter-associated bacteriuria include cues that are guidelines-discordant for CA-UTI. The understanding we gained of clinicians' mental models, especially diagnostic errors, and the algorithm developed to address these errors will inform interventions to improve the accuracy and reliability of CA-UTI diagnoses.
\end{abstract}

Keywords: Catheter-associated bacteriuria, Urinary tract infections, Evidence based guidelines, Diagnostic errors

\footnotetext{
*Correspondence: anaik@bcm.edu

${ }^{1}$ Houston Health Services Research and Development Center of Excellence,

Michael E. DeBakey VA Medical Center, Houston, TX, USA

${ }^{2}$ Department of Internal Medicine, Baylor College of Medicine, Houston, TX, USA

Full list of author information is available at the end of the article
} 


\section{Background}

Catheter-associated urinary tract infection (CA-UTI) and catheter-associated asymptomatic bacteriuria (CA-ABU) are very common yet distinct forms of catheter-associated bacteriuria [1,2]. In CA-UTI, the patient has specific urinary symptoms, and the condition merits treatment with antibiotics [1]. In contrast, $\mathrm{CA}-\mathrm{ABU}$ is marked by the absence of urinary-specific symptoms, and treatment with antibiotics does not reduce mortality, bacteremia, or subsequent risk of UTI [2]. Thus, both the Infectious Diseases Society of America (IDSA) and US Preventive Services Task Force discourage screening for, and treatment of, CA-ABU in most clinical settings [3]. Recent guidelines by IDSA provide excellent summaries of the evidence supporting these recommendations [1,2].

Despite the IDSA guidelines, inappropriate treatment of CA-ABU with antibiotics is widespread, and guidelines adoption remains modest [4]. Recent studies of CA-ABU in hospital settings show as many as $52 \%$ of patients with $\mathrm{CA}-\mathrm{ABU}$ being treated unnecessarily with antibiotics [5-9]. The cause of CA-ABU overtreatment is multifaceted and grounded in the clinical norms and inappropriately constructed mental models clinicians use to make diagnostic decisions for patients with catheterassociated bacteriuria. Conventional teaching is that the bladder and the urine within it are sterile, but this "norm" does not apply to catheterized patients in contemporary medical settings. Making the diagnosis of $\mathrm{CA}-\mathrm{ABU}$ requires the clinician to discount clinical cues, such as bacteriuria and pyuria, because neither of these can be used to distinguish between CA-ABU and CA-UTI $[1,2,10]$. Another clinical norm that runs counter to evidence is the erroneous belief that vague, non-urinary symptoms can be attributed to bacteriuria [11-14]. Additionally, clinicians often overweigh the risk of withholding antibiotics while underweighting the risk of antibiotic exposure in an individual patient [15]. These evidence-discordant norms and biases produce decision-making processes that differ in distinct and clinically important ways from evidence-based guidelines for diagnosing catheter-associated bacteriuria [16,17].

The IDSA guidelines governing catheter-associated bacteriuria are based on high-quality reviews of the available evidence [1,2]. However, the complexity and sheer length of the guidelines (51 pages) may impede their uptake $[18,19]$. Classically, diagnostic reasoning is thought to involve complex, analytical evaluations of clinical and laboratory cues to frame prior probabilities of differential diagnoses to arrive at the accurate diagnosis [20]. Empiric evidence suggests that clinicians store disease models reflecting common symptoms associated with diseases within their memory [21]; these models are called 'mental models'. These mental models are normally constructed during training when clinicians learn the symptoms associated with diseases, and are enhanced as they gain experience throughout their career. When the mental models are not accurate, diagnostic errors may occur. Use of inaccurate (guidelines-discordant) mental models associated with $\mathrm{CA}-\mathrm{ABU}$ can result in misdiagnosing CA-ABU as CA-UTI. Mental models for CA-UTI that are properly constructed (guidelinesconcordant) are reflected in Table 1 left column. A guidelines-discordant mental model for CA-UTI (commonly used when misdiagnosing CA-ABU as CA-UTI) is shown in the right column of Table 1.

In essence, prior research demonstrates that inappropriate treatment of $\mathrm{CA}-\mathrm{ABU}$ with antibiotics is widespread and guidelines adoption remains modest. To address this problem, the first objective of this research was to confirm our suspicion that clinicians' mental models are inaccurately constructed and to find the points of difference from evidence-based guidelines. The second objective was to develop a means of re-directing clinicians' mental models by creating a valid and reliable algorithm grounded in clinical evidence, with the ultimate objective of informing a guidelines implementation intervention.

\section{Methods}

We framed the problem and our approach to the problems using a two phase study approach based on the following hypotheses. First, when clinicians attempt to differentiate catheter-associated bacteriuria as either CA-UTI or CA-ABU, their mental models include both guidelines-discordant and guidelines-concordant cues resulting in (a) poor diagnostic accuracy differentiating between CA-UTI and CA-ABU (reliability with clinical guidelines and/or clinical experts) and (b) low rates of diagnostic agreement between each other (low interrater reliability among non-experts). Phase 1 evaluated the accuracy and inter-rater reliability of clinicians' mental models for catheter-associated bacteriuria. Second, we distilled the IDSA guidelines into an algorithm to attempt to improve diagnostic accuracy and inter-rater reliability by substituting guidelines-concordant cues in place of guidelines-discordant cues. In essence, the algorithm serves to recalibrate clinicians' mental models for differentiating CA-UTI from CA-ABU. Phase 2 describes the development, preliminary validation, and evaluation of inter-rater reliability of this algorithm for recalibrating clinicians' mental models. This research was conducted with the approval of the Baylor College of Medicine Internal Review Board (protocol H \#24180).

\section{Phase 1 - Clinicians' decision-making when diagnosing CA-UTI \\ Study design}

We conducted a comprehensive assessment of the diagnostic cues clinicians use when distinguishing between 


\begin{tabular}{|c|c|}
\hline $\begin{array}{l}\text { Guideline concordant signs } \\
\text { and symptoms of CAUTI }\end{array}$ & $\begin{array}{l}\text { Guideline discordant signs } \\
\text { and symptoms of CAUTI }\end{array}$ \\
\hline Fever & Pyuria (white blood cells in urine) \\
\hline Delirium & Foul smelling urine \\
\hline Rigors & Change in urine color \\
\hline Flank pain & Sediment in urine \\
\hline $\begin{array}{l}\text { Acute hematuria } \\
\text { (red blood cells in urine) }\end{array}$ & $\begin{array}{l}\text { Systemic leukocytosis (higher than } \\
\text { normal white blood cell count) }\end{array}$ \\
\hline Pelvic discomfort & Prior "UTI" diagnosis \\
\hline Urgency & Resistant organism in urine \\
\hline Frequency & Vague malaise \\
\hline Dysuria & Weakness \\
\hline Suprapubic pain & Type of organism in urine \\
\hline
\end{tabular}

CA-UTI and CA-ABU, through a case-based diagnosis exercise followed by in-depth, cognitive interviews [22].

\section{Participants}

Participants consisted of six physicians and four allied health professionals recruited from a convenience sample of experienced clinicians working in local acute and extended care facilities of a single health system. The sample of clinicians included three males and seven females with a range of 11-15 years of experience treating older patients in long-term care and inpatient settings (see Table 2). All 10 participants reviewed each of the four cases for a total of 40 cases on which analyses are based.

\section{Procedures}

Clinicians were asked to review the electronic medical records of four patients with positive urine cultures associated with an indwelling urinary catheter. Henceforth, for simplicity, we will refer to these positive urine

Table 2 Characteristics of study participants

\begin{tabular}{|c|c|c|}
\hline & $\begin{array}{l}\text { Phase } 1 \text { participants } \\
(n=10)\end{array}$ & $\begin{array}{l}\text { Phase } 2 \text { participants } \\
(n=6)\end{array}$ \\
\hline Characteristic & Number (\%) & Number (\%) \\
\hline Female gender & $7(70)$ & $4(67 \%)$ \\
\hline \multicolumn{3}{|l|}{ Occupation } \\
\hline Physician & $6(60)$ & $4(67 \%)$ \\
\hline Physician's Assistant & $2(20)$ & $1(17 \%)$ \\
\hline Nurse Practitioner & $2(20)$ & $1(17 \%)$ \\
\hline \multicolumn{3}{|c|}{ Duration of Clinical Experience } \\
\hline $1-5$ years & $1(10)$ & $4(67 \%)$ \\
\hline $6-10$ years & $3(30)$ & \\
\hline $11-15$ years & $5(50)$ & $2(33 \%)$ \\
\hline$>15$ years & $1(10)$ & \\
\hline
\end{tabular}

cultures, both bacteriuria and funguria, as "bacteriuria". All participants independently reviewed the same four urine cultures, representing four distinct cases. The selected cases were actual patient cases representing a spectrum of clinical cues and treatments representative of CA-UTI and CA-ABU. Each case presentation had at least one clinical cue shown in prior studies to influence physicians' decision-making regarding antimicrobial treatment (e.g., older age, pyuria, and type of organism) $[6,23]$. Table 3 describes the patient cases. For each of the four cases, clinicians answered two written questions: (1) Do you feel this is a CA-UTI or CA-ABU? and (2)What helped you decide if this case was a CA-UTI or CA-ABU? Subjects then underwent a cognitive interviewing exercise [22] to elicit their reasoning processes where they answered the following question: "For the case of Patient X, was your decision of CA-UTI versus CA-ABU influenced by any of the following?" Choices included pyuria (white blood cells in urine), systemic leukocytosis (white blood cells in bloodstream), type of organism in the urine (Gram negative, Gram positive or fungal), elderly or frail patient, weakness, cloudy urine, foul-smelling urine, and specific urinary symptoms (e.g. dysuria-painful urination or frequency). The researcher stated each probe one-at-a-time, after which the clinician responded with a 'yes' or 'no'. To assess use of the guidelines, clinicians were then asked "For the case of Patient X, do you feel that you applied the IDSA guidelines to arrive at your decision?" With participant consent, all interviews were tape recorded and subsequently transcribed and analyzed.

\section{Analyses for phase 1}

First, we categorized each of the signs or symptoms participants identified as influencing their decision as (1) a guidelines-concordant clinical cue for distinguishing between CA-UTI and CA-ABU, or (2) a guidelinesdiscordant clinical cue that should not be used to distinguish between CA-UTI and CA-ABU. We then calculated Fleiss' kappa to examine the overall interrater reliability of diagnoses for the four cases across all 10 clinicians, as well as the inter-rater reliability of clinicians that reported using the guidelines in their decision-making and those that reported not doing so. The MAGREE.SAS macro in SAS Version 9.2 was used to calculate the generalized kappa of Fleiss.

\section{Phase 2 - guideline-based algorithm development and validation \\ Algorithm development}

We prepared a diagnostic algorithm for catheter-associated bacteriuria based on the IDSA guidelines. This evidencebased, diagnostic algorithm was designed to improve clinicians' diagnostic ability to distinguish between CA-UTI 
Table 3 Description of bacteriuria cases diagnosed by long-term care providers and signs/symptoms endorsed

\begin{tabular}{|c|c|c|c|c|c|c|}
\hline Case & $\begin{array}{l}\text { Culture } \\
\text { results }\end{array}$ & $\begin{array}{l}\text { Diagnosis based } \\
\text { on guideline } \\
\text { criteria }\end{array}$ & Comments & $\begin{array}{l}\text { Guideline-consistent } \\
\text { diagnoses (\%) }\end{array}$ & $\begin{array}{l}\text { Guideline-concordant } \\
\text { signs/symptoms endorsed } \\
\text { (Number of clinicians) }\end{array}$ & $\begin{array}{l}\text { Guidelines-discordant } \\
\text { signs/symptoms endorsed } \\
\text { (Number of clinicians) }\end{array}$ \\
\hline \multirow[t]{5}{*}{1} & \multirow[t]{5}{*}{$\begin{array}{l}>10^{5} \mathrm{CFU} / \mathrm{mL} \\
\text { Klebsiella } \\
\text { Pneumoniae }\end{array}$} & \multirow[t]{5}{*}{$C A-A B U$} & \multirow[t]{5}{*}{$\begin{array}{l}\text { Systemic leukocytosis, } \\
\text { receiving systemic } \\
\text { corticosteroids }\end{array}$} & \multirow[t]{5}{*}{$6(60 \%)$} & $\begin{array}{l}\text { Urinary symptoms } \\
\text { incorrectly identified } \\
\text { as present (1) }\end{array}$ & Pyuria (5) \\
\hline & & & & & \multirow[t]{4}{*}{ Lack of fever (6) } & Leukocytosis (7) \\
\hline & & & & & & Elderly/frail patient (5) \\
\hline & & & & & & Weakness (3) \\
\hline & & & & & & Organism number (1) \\
\hline \multirow[t]{4}{*}{2} & \multirow[t]{4}{*}{$\begin{array}{l}<10^{4} \mathrm{CFU} / \\
\mathrm{mL} \text { gram } \\
\text { positive } \\
\text { organisms }\end{array}$} & \multirow[t]{4}{*}{ CA-UTI } & \multirow[t]{4}{*}{$\begin{array}{l}\text { Fever of } 103.3 \\
\text { degrees, and no } \\
\text { other source } \\
\text { identified }\end{array}$} & \multirow[t]{4}{*}{$4(40 \%)$} & $\begin{array}{l}\text { Lack of urinary } \\
\text { symptoms (2) }\end{array}$ & Leukocytosis (4) \\
\hline & & & & & Fever (6) & Organism type (3) \\
\hline & & & & & Delirium (3) & Elderly/frail patient (5) \\
\hline & & & & & Hematuria (3) & Isolated Organisms (3) \\
\hline \multirow[t]{7}{*}{3} & \multirow{7}{*}{$\begin{array}{l}>10^{5} \mathrm{CFU} / \\
\mathrm{mL} \text { E. coli; } \\
\text { and }>10^{3}- \\
<10^{5} \mathrm{CFU} / \\
\mathrm{mL} \\
\text { Klebsiella } \\
\text { oxytoca }\end{array}$} & \multirow[t]{7}{*}{$C A-A B U$} & \multirow{7}{*}{$\begin{array}{l}\text { Leg weakness, no } \\
\text { symptoms of urinary } \\
\text { tract infection }\end{array}$} & \multirow[t]{7}{*}{$1(10 \%)$} & & Pyuria (3) \\
\hline & & & & & & Leukocytosis (7) \\
\hline & & & & & & Organism type (6) \\
\hline & & & & & & Elderly/frail patient (5) \\
\hline & & & & & & Weakness (6) \\
\hline & & & & & & Patient fall (5) \\
\hline & & & & & & History of UTIs (3) \\
\hline \multirow[t]{5}{*}{4} & \multirow[t]{4}{*}{$\begin{array}{l}>10^{5} \mathrm{CFU/} \\
\mathrm{mL} \text { Candida } \\
\text { albicans }\end{array}$} & \multirow[t]{4}{*}{$C A-A B U$} & \multirow[t]{4}{*}{$\begin{array}{l}\text { No symptoms of } \\
\text { urinary tract infection }\end{array}$} & \multirow[t]{4}{*}{$10(100 \%)$} & $\begin{array}{l}\text { Presence of respiratory } \\
\text { symptoms } \\
\text { [alternate cause] (4) }\end{array}$ & $\begin{array}{l}\text { Lack of leukocytosis/mild } \\
\text { Leukocytosis (3) }\end{array}$ \\
\hline & & & & & Lack of fever (3) & Organism type (6) \\
\hline & & & & & Intact mental status (2) & $\begin{array}{l}\text { Elderly/frail patient } \\
\text { [likely to colonized } \\
\text { candida] (3) }\end{array}$ \\
\hline & & & & & No urinary symptoms (2) & \\
\hline & Total & & & $21(53 \%)$ & & $\begin{array}{l}\text { Leukocytosis, pyuria, } \\
\text { frailty cited by } 3-7 \\
\text { respondents in every case }\end{array}$ \\
\hline
\end{tabular}

$C A-A B U=$ catheter associated asymptomatic bacteriuria, $C A-U T I$ catheter associated urinary tract infection.

and $\mathrm{CA}-\mathrm{ABU}$. The first version of the algorithm, developed according to the 2005 IDSA guidelines on CA-ABU and the 2009 IDSA guidelines on CA-UTI [1,2], was formatted as a flowchart to fit onto a pocket-sized card for high portability. The algorithm was evaluated for content and face validity, and revisions were made accordingly after each evaluation (see below for details). The final version of the algorithm was then used by three trained research personnel to classify cases of bacteriuria as either CA-UTI or CA-ABU (See Figure 1).

\section{Algorithm validation: content validity}

To examine content validity of the algorithm, the initial version was distributed via email to all 11 expert panel members of the IDSA CA-UTI and CA-ABU guidelines committee. In a cover letter to our email message, we asked three survey questions (see Table 4 for specific wording); each question maps to one of the following standards: criterion, diagnostic, and feasibility. The response to each question was scored on a 10-point scale, with higher numbers indicating stronger agreement. We also provided a space for respondents to make comments or suggestions regarding the algorithm. Seven (64\%) panel members responded with a numeric score, while one additional panel member provided comments only. The mean score for each question and each respondent was computed, and comments were tabulated for review by our research team.

\section{Algorithm validation: face validity Design and participants}

We conducted cognitive interviews with six non-expert clinicians recruited from a purposive sample of clinicians 
working in local acute and extended care facilities to evaluate face validity of the algorithm. These participants, four internal medicine resident physicians, one nurse practitioner, and one physician assistant were chosen because all routinely provide care for catheterized adults and thus would be potential users of the algorithm.

\section{Procedures}

Participants were asked the following question regarding each step of the algorithm: "As you look at this diamond (decision point) or box (procedure step), what are you thinking it refers to?" Cognitive interview responses were categorized based on whether there was a misunderstanding, wrong interpretation, over-interpretation,

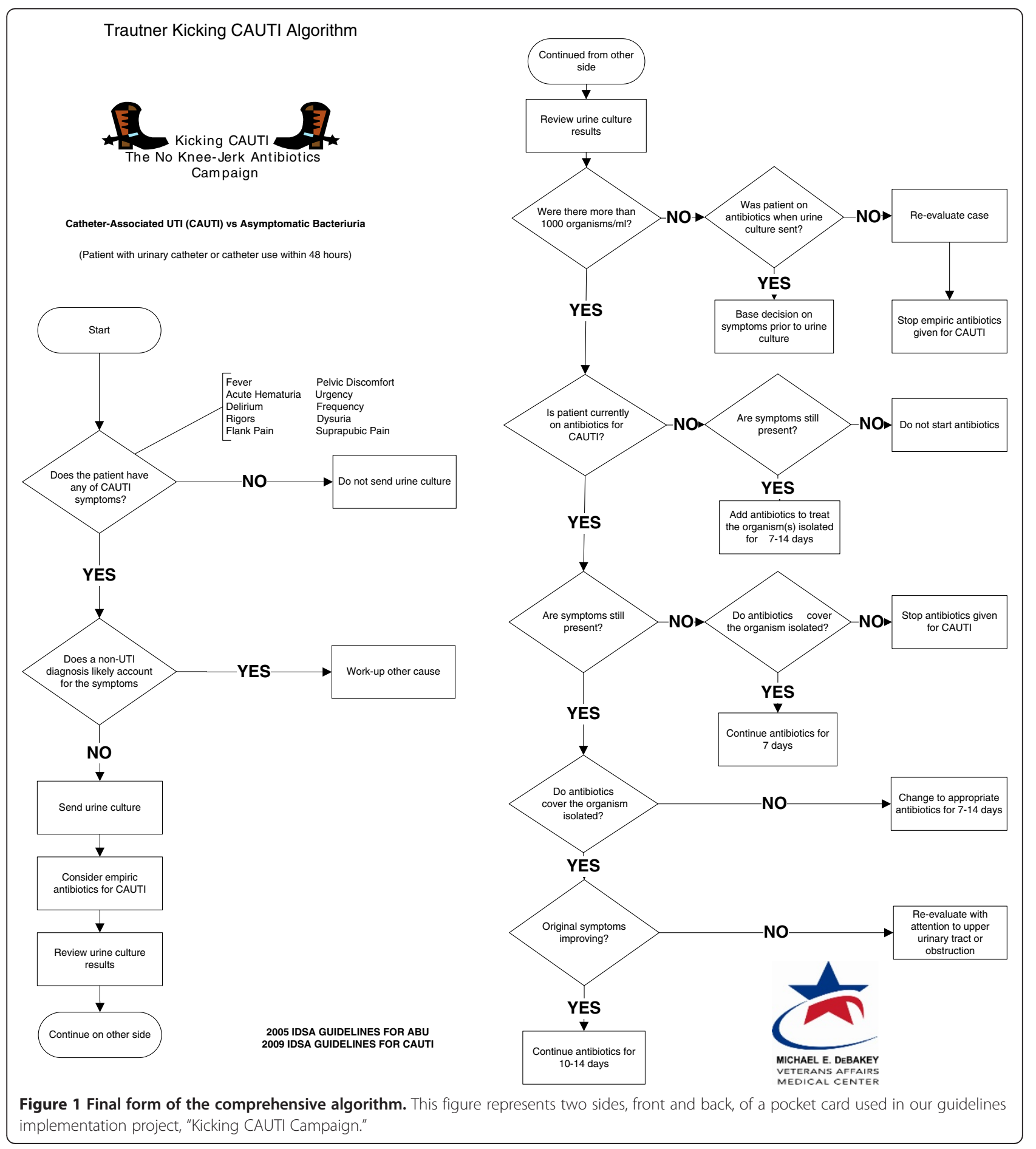


correct interpretation, or off-topic response to each step of the algorithm [22]. The number of responses in each category was tallied, and percentage of total responses falling into each category was calculated. Based on the answers given, the algorithm was further revised to its ultimate form. This final version (see Figure 1) was sent back to the lead authors of the relevant IDSA guidelines $[1,2]$ for their final input; neither suggested any substantial changes.

\section{Algorithm reliability: inter-rater reliability between non-experts and clinical expert}

In order to determine if use of a guideline concordant algorithm has the potential to recalibrate inaccurately built mental models resulting in improved diagnostic accuracy of CA-UTI and CA-ABU, one expert and two non-expert providers used the algorithm to classify 71 distinct cases of catheter-associated bacteriuria arising in our local acute and extended care facilities as either CA-UTI or CA-ABU. Reliability ratings between nonexpert and expert raters were used to confirm the ability of the algorithm to improve diagnostic accuracy. We also calculated inter-rater reliability of the algorithm between ratings of the two non-experts. Cases were chosen consecutively at 3-4 month intervals over a 10 month period. Each case was classified independently by the expert and at least one non-expert, resulting in 110 paired comparisons (as 20 cases were rated by the expert and both non-experts). Non-expert raters were trained to use the algorithm in introductory exercises prior to performing Cohen's simple kappa the case classifications. Following procedures identical to those we used in Phase 1, the three raters were each given full access to the patients' medical records including dates of the relevant urine cultures. Raters classified each case independently, and each rater was blinded to the other raters' classifications. Because we were interested in the interrater reliability between specific pairs of raters, Cohen's simple kappa was used to examine inter-rater reliability of accurate diagnoses aided by the algorithm between the expert and each non-expert and explore inter-rater reliability of the algorithm between non-experts [24].

\section{Results}

Phase 1- clinicians' decision-making when diagnosing CA-UTI

Twenty-one (53\%) of the 40 bacteriuria diagnoses by study participants were guideline-concordant (Table 3). Furthermore, only seven of the ten clinicians said that they applied the IDSA guidelines to arrive at their decisions; the other three said they did not use the guidelines by intention or they had not read the guidelines.

In terms of guideline-concordant clinical cues, six clinicians consistently identified fever as a guidelineconcordant cue for CA-UTI (Table 3), and two clinicians commented on the presence or absence of urinary symptoms as being influential in their decision-making. On any given case, guideline-discordant cues (Table 1, right column) were endorsed by five or more clinicians when attempting to distinguish CA-ABU from CA-UTI.

All ten clinicians correctly identified case 4 as CA-ABU (Table 3), but some for the wrong reasons, as six reported that the organism type (Candida) influenced their decision, and three reported that the lack of leukocytosis influenced their decision. Interestingly, three clinicians also cited the presence of a chronic catheter in an elderly patient as a significant factor in their decision. This sign lead them to think that the patient was more likely to be colonized with a fungal agent. In Table 3 case 3 , only one clinician correctly identified this case as a CA-ABU as the others were misled by guideline-discordant cues, e.g., leukocytosis and weakness in the patient. In Table 3 case 2 , six clinicians correctly identified fever as a clinical cue for CA-UTI, but only four clinicians concluded that the patient had CA-UTI. Additionally, three clinicians each endorsed organism type and low number of organisms as leading them to conclude the patient had $\mathrm{CA}-\mathrm{ABU}$. In essence, clinicians could not distinguish which of these cues were guideline-concordant for CA-UTI. For Table 3 case 1 , six clinicians arrived at the diagnosis of $\mathrm{CA}-\mathrm{ABU}$, but seven reported the guideline-discordant cues of leukocytosis as influential in their decision making. In this case, the patient was on oral steroids and therefore had an alternative

Table 4 Ratings of the diagnostic algorithm by expert members of the Infectious Diseases Society of America (IDSA) guidelines panel for diagnosing and managing catheter associated bacteriuria

\begin{tabular}{lccc}
\hline & $\begin{array}{c}\text { Content meets } \\
\text { Criterion standard }\end{array}$ & $\begin{array}{c}\text { Content meets } \\
\text { Diagnostic standard }\end{array}$ & $\begin{array}{c}\text { Content meets } \\
\text { feasibility standard }\end{array}$ \\
\hline $\begin{array}{l}\text { Questions posed to IDSA } \\
\text { guidelines panel members }\end{array}$ & $\begin{array}{c}\text { Does the algorithm appropriately } \\
\text { reflect the definition of CA-UTI as } \\
\text { per the IDSA guidelines? }\end{array}$ & $\begin{array}{c}\text { Does the algorithm reflect an appropriate } \\
\text { approach to diagnosis and treatment of } \\
\text { CAUTI vs. ABU, as recommended by the }\end{array}$ & $\begin{array}{c}\text { Could you apply this } \\
\text { algorithm to your own } \\
\text { catheterized inpatients? }\end{array}$ \\
$\begin{array}{llll}\text { Experts' ratings, mean } \\
\text { (standard deviation) }\end{array}$ & $8.1(s d=1.1)$ & $7.1(s d=1.2)$ & $8.0(s d=1.6)$ \\
\hline
\end{tabular}

$(\mathrm{N}=7)$.

Ratings were based on 10-point numerical scale with higher numbers indicating stronger agreement. 
explanation for his leukocytosis. The IDSA guidelines explicitly state that leukocytosis is not a reliable clinical cue for CA-UTI [1,2].

Inter-rater reliability among all 10 clinicians was fair (Fleiss' kappa $=0.35,95 \%$ CIs $=0.21$ and 0.50) [24]. Inter-rater reliability among the seven clinicians reporting that they used the guidelines was also fair (Fleiss' kappa $=0.28,95 \% \mathrm{CIs}=0.07$ and 0.50). Interrater reliability among the three clinicians reporting that they did not use the guidelines was substantial (Fleiss' kappa $=0.63,95 \% \mathrm{CIs}=0.06$ and 1.00), i.e., they arrived at the same diagnosis, but these diagnoses were not always guideline-concordant [24]. Therefore, despite the higher reliability rating, these clinicians' mental modes resulted in poorer diagnostic accuracy.

\section{Phase 2 - guideline-based algorithm development and validation \\ Content validity}

Eight (73\%) of the 11 members of the IDSA guidelines committee responded to our request for comments on the original algorithm. We received 27 specific comments addressing about half of the processes (boxes) or decision points (diamonds) in the algorithm. Ten (37\%) of these overall comments concerned changing the recommended duration of treatment to reflect the patient's response to therapy; we had misinterpreted this point in the guidelines. Seven of 11 members of the IDSA guidelines committee scored the algorithm along three standards (criterion, diagnostic, and feasibility) for measuring the quality of the algorithm content. Table 4 provides the mean ratings provided by IDSA guidelines committee members for each standard with each of the standards having an acceptable mean rating between 7.1 and 8.1. We modified the algorithm format to fit standard flow-charting in response to specific suggestions.

\section{Face validity}

Non-expert clinicians (see second portion of Table 2) reviewed the algorithm. Cognitive interviews with six clinicians produced comments for a total of 164 distinct comments about processes (boxes) or decision points (diamonds) contained within the algorithm. For 123 (75\%) of the comments, respondents correctly interpreted the meaning of the process or decision point of the algorithm. Eighteen $(11 \%)$ of the responses were incorrect, 13 (8\%) were over-interpreted, 7 (4\%) were misunderstood and 3 (2\%) responses were off-topic. An example of over-interpretation was a box that stated "work up another cause," which was interpreted as "do a chest $\mathrm{x}$-ray and obtain an abdominal film." The diamond that received the greatest number of incorrect responses was originally worded "Bacteriuria $\geq 10^{3} \mathrm{CFU} / \mathrm{ml}$ ?". We subsequently modified the text to read "Were there more than 1000 organisms $/ \mathrm{ml}$ ?" to reduce the need for real-time mathematical transformations and make interpretation unambiguous. The algorithm was modified to address the issues raised in the cognitive interviews, leading to its final form (see Figure 1), as approved by the two lead guidelines authors.

\section{Reliability of the algorithm for diagnostic accuracy and inter-rater reliability}

Using the final version of the algorithm, three providers classified 71 cases of catheter-associated bacteriuria. Of these cases, 28 were CA-UTI and 42 were CA-ABU as per the IDSA definitions for these conditions. Forty-nine cases were rated by both the expert and non-expert \#1, forty-one were rated by both the expert and non-expert $\# 2$, and twenty cases were rated by both non-expert $\# 1$ and non-expert \#2. Inter-rater reliability between the expert and non-expert \#1 was substantial (Cohen's kappa $=0.72,95 \%$ CIs $=0.52$ and 0.93). Inter-rater reliability between the expert and non-expert \#2 was almost perfect (Cohen's kappa $=0.80,95 \%$ CIs $=0.61$ and 0.99). The average inter-rater reliability among the expert and both non-experts was substantial (average Cohen's kappa $=0.76$ ), suggesting improved diagnostic accuracy among non-experts with the clinical expert (criterion standard). Inter-rater reliability between the two nonexperts was also substantial (Cohen's kappa $=0.88,95 \%$ CIs $=0.64$ and 1.00) [24].

\section{Discussion}

Our data show that clinicians who routinely care for patients with urinary catheters use mental models that are often guidelines-discordant when classifying cases of catheter-associated bacteriuria as either CA-UTI or $\mathrm{CA}-\mathrm{ABU}$. Their decision cues consist of a heterogeneous group of signs and symptoms, many of which are not supported by evidence or run counter to evidence, as per IDSA guidelines [1,2]. The low level of accuracy and reliability of these clinicians' diagnoses underscores the need for recalibrating their mental models to be compatible with evidence as documented in the IDSA guidelines for catheter-associated bacteriuria.

To address this need, we developed and validated an algorithm to enhance adoption of IDSA guidelines into diagnostic decisions for catheter-associated bacteriuria. A comprehensive version of the algorithm was created by mapping key decision points outlined in the CA-UTI and CA-ABU guidelines. Expert members of the IDSA guidelines panels provided content validation of the comprehensive algorithm with ratings along a 10-point scale for criterion, diagnostic, and feasibility standards. Cognitive interviews further established the face validity and usability of the comprehensive algorithm. From these results, we revised the algorithm. Finally, we 
established the reliability of the algorithm for accurately diagnosing cases as CA-UTI versus CA-ABU between expert and non-expert users and the inter-rater reliability of the algorithm between two non-expert users. High reliability between the clinical expert and each nonexpert suggests improvement in diagnostic accuracy aided by the algorithm.

The results of the current study build on previous work that demonstrates physicians are more likely to treat bacteriuria with antibiotics (and therefore assume that the patient has a UTI) when patients have clinical cues that are consistent with prior diagnostic norms and practice (e.g., bacterial as opposed to fungal infection, higher white blood cell counts in the urine, positive urine nitrites, or a change in vague behaviors from baseline) $[6,12,23]$. The use of such guidelines-discordant cues leads to the inappropriate antimicrobial treatment of CA-ABU, and, as seen in our study, inconsistent (i.e., poor inter-rater reliability) and inaccurate diagnostic decision-making among clinicians. These guidelinediscordant signs and symptoms are present within mental models that clinicians use to make diagnostic and treatment decisions [25].

Valid mental models built on prototypical cues (guideline-concordant signs or symptoms in Table 1) for CA-UTI can help to differentiate CA-UTI from CA-ABU among patients with catheter-associated bacteriuria. However, when these mental models are incorrectly constructed using cues that do not have high predictive validity (e.g., pyuria, and other guidelinediscordant symptoms) or cannot help to differentiate the two subgroups (e.g., bacteriuria is present in both CA-UTI and CA-ABU), poor diagnostic accuracy and reliability will be the result.

The diagnosis and management of catheter-associated bacteriuria can be improved through the recalibration of clinicians' mental models so that they are concordant with IDSA guidelines for differentiating CA-UTI from CA-ABU. This recalibration requires mindfulness of the guideline-discordant cues clinicians use when making diagnostic errors and substitution of guideline-concordant cues. Our study is consistent with prior evidence suggesting that simple methods, such as the use of checklists, algorithms, or protocols, combined with interventions such as audit and feedback, can enhance guideline adoption [26-28].

The current study has several limitations. Participants in our sample are not representative of clinicians from all fields of medicine, but they do reflect a group of clinicians (physicians and allied health providers) who regularly manage catheter-associated bacteriuria in a typical inpatient setting. Another limitation is that the two nonexperts were trained by an infectious disease expert who was very familiar with the guidelines content and how to use the algorithm. However, this training process is reflective of how algorithms are often implemented in real-world setting. Indeed, we are currently studying case-based audit and feedback as a focused training method for using our algorithm to decrease inappropriate use of antibiotics for CA-ABU [28]. Another limitation is the modest sample size in Phase 1; however, the number of case pairs in Phase 1 was sufficient for reliability testing. Finally, the 10 cases were chosen to be "difficult" in that each challenged clinical norms, thus likely magnifying the disagreement between the clinician's diagnosis and the guidelines-concordant diagnoses. Case classification, albeit time-consuming, is a fundamental first step for any quality improvement project related to CA-UTI. The algorithm developed in this study may improve the efficiency and reliability of case classification.

\section{Conclusions}

During the diagnostic process, clinicians commonly compare patient's symptoms to previously constructed mental models associating signs and symptoms to diseases. We have shown that use of improperly constructed (guideline discordant) mental models may result in diagnostic errors. Guidelines serve many of the same functions as mental models, in that they help identify data that are relevant to a particular diagnosis and exclude irrelevant data. The length and complexity of many guidelines limit their feasible dissemination and adoption in busy clinical settings. We have also shown that algorithms that simplify guidelines to better support decision-making in medical settings may help physicians identify and recalibrate inaccurate mental models, move toward more evidence-concordant diagnostic decisions, and reduce diagnostic errors.

\section{Competing interests}

The authors declare that they have no competing interests.

\section{Authors' contributions}

BT conceived of the study, participated in its design, drafted the manuscript and had final approval of the version submitted to the journal. RB participated in study design, obtained data and contributed to interpretation, and helped draft the manuscript. AA conducted statistical analysis and data interpretation, and helped draft the manuscript. SH participated in study design, contributed to interpretation of data, and revised manuscript critically. AG obtained data and contributed to interpretation. PK participated in study design, contributed to interpretation of data, and revised manuscript critically. VP contributed to interpretation of data and manuscript revision. AN participated in study conception and design, helped draft the manuscript, and had final approval of the version submitted to the journal. All authors read and approved the final manuscript.

\section{Acknowledgements}

This work was supported by a grant from the Veterans Administration, Health Services Research and Development Program (IIR 09-104, Trautner PI) and with resources and use of facilities at the Houston VA HSR\&D Center of Excellence (HFP90-020) at the Michael E. DeBakey VA. Dr. Trautner received support from a VA Rehabilitation Research and Development career development award (B4623). Dr. Payne receives support from the Office of 
Academic Affiliations, post-doctoral fellowship program in Health Services Research. Dr. Naik received additional support from a Doris Duke Charitable Foundation Clinical Scientist Development Award.

\section{Author details}

'Houston Health Services Research and Development Center of Excellence, Michael E. DeBakey VA Medical Center, Houston, TX, USA. ${ }^{2}$ Department of Internal Medicine, Baylor College of Medicine, Houston, TX, USA.

${ }^{3}$ Department of Internal Medicine, Tulane University School of Medicine, New Orleans, LA, USA. ${ }^{4}$ Michael E. DeBakey VA Medical Center (152), 2002 Holcombe Boulevard, Houston, TX 77030, USA.

Received: 3 August 2012 Accepted: 4 April 2013

Published: 15 April 2013

\section{References}

1. Hooton TM, Bradley SF, Cardenas DD, et al: Diagnosis, prevention, and treatment of catheter-associated urinary tract infection in adults: 2009 international practice guidelines from the Infectious Disease Society of America. Clinical Infectious Disease 2010, 50(5):625-663.

2. Nicolle LE, Bradley S, Colgan R, Rice JC, Schaeffer AJ, Hooton TM: Infectious Diseases Society of America guidelines for the diagnosis and treatment of asymptomatic bacteriuria in adults. Clin Infect Dis 2005, 40(5):643-654.

3. Lin K, Fajardo K: Screening for asymptomatic bacteriuria in adults: evidence for the U.S. Preventative Services Task Force reaffirmation recommendation statement. Ann Intern Med 2008, 149:W20-4.

4. Gross PA, Patel B: Reducing antibiotic overuse: A call for national performance measure for not treating asymptomatic bacteriuria. Clin Infect Dis 2007, 45(10):1335-1337.

5. Enterococcal bacteriuria is often overtreated and rarely leads to infectious complications. Boston: the 49th Annual Meeting of the Infectious Diseases Society of America; 2011

6. Cope M, Cevallos ME, Cadle RM, Darouiche RO, Musher DM, Trautner BW: Inappropriate treatment of catheter-associated asymptomatic bacteriuria in a tertiary care hospital. Clin Infect Dis 2009, 48:1182-8.

7. Dalen DM, Zvonar RK, Jessamine PG: An evaluation of the management of asymptomatic catheter-associated bacteriuria and candiduria at The Ottawa Hospital. Can J Infect Dis Med Microbiol 2005, 16:166-170

8. Gandhi T, Flanders SA, Markovitz E, Saint S, Kaul DR: Importance of urinary tract infection to antibiotic use among hospitalized patients.

Infect Control Hosp Epidemiol 2009, 30:193-5.

9. Khawcharoenporn T, Vasoo S, Ward E, Singh K: Abnormal urinalysis finding triggered antibiotic prescription for asymptomatic bacteriuria in the ED. Am J Emerg Med 2011, 29(7):828-830.

10. Tambyah PA, Maki DG: The relationship between pyuria and infection in patients with indwelling urinary catheters: a prospective study of 761 patients. Arch Intern Med 2000, 160:673-7.

11. Silver SA, Baille L, Simor AE: Positive urine cultures: A major cause of inappropriate antimicrobial use in hospitals? Canadian Journal of Infectious Diseases and Medical Microbiology 2009, 20(4):107-111.

12. Walker S, McGreer A, Simor AE, Armstrong E, Loeb M: Why are antibiotics prescribed for asymptomatic bacteriuria in institutionalized elderly people? A qualitative study of physicians' and nurses' perceptions. CMAJ 2000, 163(3):273-277.

13. Drinka PJ, Crnich CJ: Diagnostic accuracy of criteria for urinary tract infection in a cohort of nursing home residents. J Am Geriatr Soc 2008, 56:376-7.

14. Sundvall PD, Ulleryd P, Gunnarsson RK: urine culture doubtful in determining etiology of diffuse symptoms among elderly individuals: a cross-sectional study of 32 nursing homes. BMC Fam Pract 2011, 12:36.

15. Powers JH: Risk perception and inappropriate antimicrobial use: yes, it can hurt. Clin Infect Dis 2009, 48:1350-3.

16. Kahneman D, Fredrick S: Representativeness revisited: Attribute substitution in intuitive judgment. In Heuristics and biases: The psychology of intuitive judgment. Edited by Gilovich T, Griffin D, Kahneman D. Cambridge, UK: Cambridge University Press; 2002:49-81.

17. Cavazos JM, Naik AD, Woofter A, Abraham NS: Barriers to physician adherence to nonsteroidal anti-inflammatory drug guidelines: A qualitative study. Aliment Pharmacol Ther 2008, 28:789-798.
18. Grol R, Grimshaw J: From best evidence to best practice: effective implementation of change in patients' care. Lancet 2003, 362:1225-1230.

19. Foy R, Eccles MP, Jamtvedt G, Young J, Grimshaw JM, Baker R: What do we know about how to do audit and feedback? Pitfalls in applying evidence from a systematic review. BMC Health Services Research 2005, 5:50.

20. Sox HC, Blatt MA, Higgins MC, Marton Kl: Medical Decision Making. 2nd edition. Philadelphia: American College of Physicians Press; 2007.

21. Gigerenzer G, Gaissmaier W: Heuristic Decision Making. Annu Rev Psychol 2011, 62:451-482.

22. Cognitive Interviewing: A How-to Guide. Short Course presented at the 1999 Meeting of the American Statistical Association. Durham, NC: Research Triangle Institute; 1999.

23. Chant C, Dos Santos CC, Saccucci P, Smith OM, Marshall JC, Friedrich JO: Discordance between perception and treatment practices associated with intensive care unit-acquired bacteriuria and funguria: A Canadian physician survey. Crit Care Med 2008, 36(4):1158-1166.

24. Landis JR, Koch GG: The measurement of observer agreement for categorical data. Biometrics 1977, 33(1):159-174.

25. Wegwarth O, Gaissmaier W, Gigerenzer G: Smart heuristics for doctors and doctors-in-training: heuristics in medicine. Medical Education 2009, 43:721-728

26. Cahill NE, Suurdt J, Ouellette-Kuntz H, Heyland DK: Understanding adherence to guidelines in the intensive care unit. Development of a comprehensive framework. J Parenter Enteral Nutr 2010, 34(6):616-624

27. Loeb M, Brazil K, Lohfield L, McGreer A, Simor A, et al: Effect of a multifaceted intervention on number of antimicrobial prescriptions for suspected urinary tract infections in residents of nursing homes: Cluster randomized control trial. BMJ 2005, 331(7518):669.

28. Trautner BW, Kelly PA, Petersen N, Hysong S, Kell H, et al: A hospital-site controlled intervention using audit and feedback to implement guidelines concerning inappropriate treatment of catheter-associated asymptomatic bacteriuria. Implement Sci 2011, 6(1):41.

doi:10.1186/1472-6947-13-48

Cite this article as: Trautner et al.: Development and validation of an algorithm to recalibrate mental models and reduce diagnostic errors associated with catheter-associated bacteriuria. BMC Medical Informatics and Decision Making 2013 13:48.

\section{Submit your next manuscript to BioMed Central and take full advantage of:}

- Convenient online submission

- Thorough peer review

- No space constraints or color figure charges

- Immediate publication on acceptance

- Inclusion in PubMed, CAS, Scopus and Google Scholar

- Research which is freely available for redistribution
() Biomed Central 